\title{
To the heart of cardiac injury
}

Jana Koth is classic biologist, interested in cardiac repair in zebrafish at the Weatherall Institute of Molecular Medicine at Oxford University. Adult zebrafish, she explains, have an ability that humans and adult mammalian model systems do not: they can regenerate their heart following injury. But unlike larval and embryonic forms, adult zebrafish are naturally opaque, with limited options to detail changes over time. Histology and antibody staining of heart sections have been important tools, but are destructive by nature. Koth thinks the inability to easily image changes in living animals has been an impediment to the field in the past and held back the potential of adult zebrafish as a model of regeneration.

To see into to the living heart, Koth approached Jürgen Schneider, a magnetic resonance physicist at Oxford, to ask an interesting question of magnetic resonance technology: can we image a tiny fish?

"We are very familiar with maintaining rats and mice in the magnet," Schneider says; his own research uses magnetic resonance imaging (MRI) and magnetic resonance spectroscopy to characterize and phenotype rodent models of cardiovascular disease. "But how on earth do you maintain a fish in a magnet?" he says, recalling the challenges they faced in adapting the MRI setup to accommodate a fish in water without flooding the machine.

But maintaining the fish in physiologically stable conditions was an important consideration for Koth. She explains that other researchers have used techniques like tomography or ultrasound to record cardiac injury in living zebrafish before, but that these approaches involve taking the fish out of the water and placing it on its back in a damp sponge for the procedure. This is stressful, she says, and cannot be sustained for very long. Ultrasound is also limited in its ability to capture tissue detail like MRI can, Schneider adds.

So the teams sat down to figure out their MRI approach, a collaboration of basic biology meeting sophisticated imaging, says Schneider. "Without the interdisciplinary efforts, the entire project would not have gone anywhere." Their results and methodology can be found in Scientific Reports ( 7 , 2917; 2017).

Schneider and his team started by imaging a fixed fish using a $13 \mathrm{~mm} R F$ coil, the

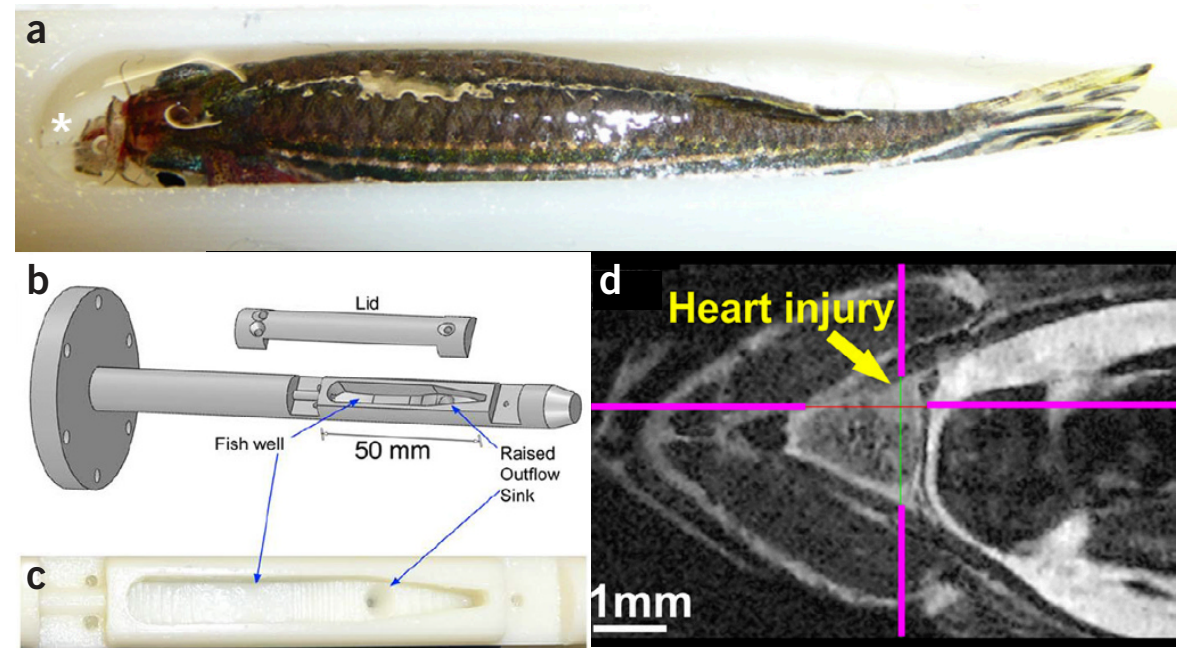

3D printed chamber designed to hold an adult zebrafish during MRI (a-c) and an MR image showing heart lesion two days after an injury (d). Adapted from Sci. Rep. 7, 2917 (2017).

smallest coil the lab had available that had been used in the past for studying mouse embryos. After confirming the level of detail possible, it was then a matter of setting up the infrastructure to hold a living fish.

Koth sketched out setups that would keep a half gram zebrafish alive and anesthetized for extended periods of time, within the constraints needed to capture a highresolution image. The resulting design was a 3D-printed chamber with a small well to hold the animal upright while gently perfusing it via an inflow tube with aerated water and anesthetic. With the device built and an imaging contrast agent injected, the team established that two 15-minute scans could create sufficiently high resolution images while minimizing the time the fish spent in the chamber. After the scan, fish recovered within a few minutes of being placed in an anesthetic-free tank.

In the initial results reported, they were able to distinguish damaged cardiac tissue in injured fish from that of healthy animals and follow repair over several weeks post-injury.

Notably, Schneider says, no fish died during testing-even those with induced cardiac injuries-allowing repeated recordings. "There is a demand for looking at the same fish over time which hasn't been met before," says Koth, "which we've tried to fill with our method." She continues, "Particularly with cancers or injuries, these tissue alterations are all unique and you want to follow each single one over time and profile the tissue changes or repair." Though histology and antibody staining won't be replaced by the MRI technique, it should be an important additional tool. "There are issues which you cannot overcome by looking at different individuals at different time points after a treatment, procedure, or injury," Koth says.

Thinking of how a zebrafish-compatible MRI will prove useful for their own research, Schneider thinks of characterizing how the heart beats. "This will take the development to the next level," he says. "Whether or not it's going to be feasible to quantify cardiac function in the zebrafish analogously to the mouse heart or to the human heart remains to be seen, but that would certainly be something I would be interested in as a next step."

Koth is also ready for some manipulations. She would like to see whether her lab can enhance or hinder repair by modulating different gene expressions and pathways of interest. "We would like to follow up with the MRI technique to show that we can, over time, influence the repair rate with our interventions," she says.

Theoretically, access to a preclinical MRI machine and a 3D printer-both are increasingly available, at least at larger universitiescould get other researchers on their way to their own recordings; Schneider and Koth are happy to collaborate and offer some of their expertise to those interested.

\section{Ellen P. Neff}

